\title{
Riesgos de recaídas en niños con epilepsia después de suspender medicamentos anticonvulsivos
}

\author{
Dr. Marcelo Devilat B. ${ }^{1}$; Dr. Bolívar Valenzuela M. ${ }^{1}$; Dr. Juan Enrique Blumel M.1.2 \\ Risk factors of seizures recurrences after withdrawal of \\ anticonvulsant drugs in epileptic children
}

To determine the frequency of recurrences and the factors which may influence it, 61 epileptic children previously treated for 5 seizur e free years and followed-up for at least 2 years after discontinuation of drugs were studied. In $29.5 \%$ of the patients recurrences were detected, in the majority of them within the first two years after drug discontinuation. Recurtences were significantly related to poor evolution during treatment, use of 2 ar more anticonvulsants and history of one or more drug treatment periods before the one under analysis. There were no significant relationships of recurrences with sex, family epilepsy, type of seizure, age, initiation of the efinical picture nor number of seizures previous to treatment.

(Key words: Epilepsy, treatment withdrawal, recurrences, frequency, type of treatment, number of drugs, sex, Tamily history).

Llamamos epilepsia a la presentación de dos o más crisis epilépticas afebriles separadas al menos por 24 horas entre sí ${ }^{1,2}$. Debe ser diferenciada de las crisis epilépticas únicas ${ }^{3}$ y de la oligoepilepsia ${ }^{4}$. Aunque su tratamiento es prolongado. costoso y no exento de efectos colaterales inde. seables ${ }^{5}$, no hay acuerdo entre los especialistas acerca de cuánto tiempo deben ser medicados los pacientes para obtener remisión de ella, ní tam. poco el periodo de observación a que deben ser sometidos una vez retirados los anticonvulsivantes $^{6}$.

El objetivo de esta investigación fue medir la frecuencia de remisiones totales y recaídas en un grupo de 61 niños con epilepsia, así como identificar factores que incidan en las recaídas.

\section{MATERLAL Y METODOS}

Entre 1977 y 1978, a 205 niños tratados por crisis epilépticas, que habian estado libres de dichos episodios por 5 años, se les suspendió la medicación gradualmente en un año. Noventa $y$ un pacientes pudieron ser observados por lo menos 2 af̃os sin anticonvuisivantes 6,7 . De éstos, 61 tenían epilepsia y son los que componen la muestra estudiada.

El grupo estaba formado por 35 varones y 26 niñas,

1. Servicio de Neurología y Siquiatria infantil, Hospital Luis Calvo Mackenta. División Ciencias Médicas Oriente. Facultad de Medjcina. Universidad de Chile.

2. Computación. Laboratorio Central, Hospital Bartos Luco-Truddeau. División Ciencias Médicas Sur. Facultad de Medicina. Universidad de Chile. quienes habían iniciado su enfermedad entre los 3 méses y los 11 años de vida $(\bar{x}=2,47 ; D E \pm 2,3$ años). La edad promedio al comenzar el tratamiento fue de 3,64 años, (DE $\pm 2,25$ años)

Cincuenta y dos enfermos $(85,2 \%)$ habían tenido crisis generalizadas (tónico-clónicas, tónicas, atónicas) 1 de ellos presentaba ausencirs simples; 9 niños $(14,8 \%)$ sufrían crisis parciales (simples o complejas), 6 de los cuales tuvieron en alguna oportunidad una crisis generalizada.

Cuarenta y cinco pacientes $(73,8 \%$ padecían epilepsia idiopática, en tanto que $15(24,6 \%)$ tuvieron algún tipo de afección pre-periodo postnatal. En uno $(1,6 \%)$ no se pudo obtener información al respecto. El examen neurológico fue anormal en $5(8,2 \%)$. Igual número presentaba retardo mental $(C l<70)$. Treinta enfermos $(49,2 \%)$ tenían a gún pariente de primero o segundo grado con epilepsia. Cuarenta $y$ nueve niños $(80,3 \%)$ recibieron el tratamiento con un sólo fármaco y 12 $(19,7 \%)$ con 2 ó 3 , debido a recaídas durante el tratamiento original. Los medicamentos más usados fueron fenobarbital y fenjtoína, en dosis de $5 \mathrm{mg} x \mathrm{~kg} x$ día.

El tiempo total de tratamiento de los pacientes que no recayeron, incluyendo el período de suspensión gradual del fármaco, fue en promedio de 6,31 años (DE $\pm 1,91$ ). El perído total de observación sin medicamentos fue de 3,03 años (DE $\pm 1,72$ ) y el tiempo total de tratamiento $y$ observación fue de 9,54 años (DE \pm 1,87 ).

El análisis estadístico se hizo mediante ha pruebas de Chi-cuadrado y de Fisher con la corrección de Yates, cuando su uso fue necesario, Los valores significativos fueron referidos a $\mathrm{p}<0,05$.

\section{RESULTADOS}

Dieciocho niños $(29,5 \%)$ recayeron, después de por lo menos 2 años de observación sin 
medjcamentos: $5(27,7 \%)$ durante el periodo de retiro gradual del fármaco; (4 en los primeros cuatro meses). Las recaídas de los restantes 13 enfermos $(72,3 \%)$ ocurrieron después de la suspensión total de los medicamentos: en $8(61,5 \%)$ durante el primer año de observación, en 4 $(30,8 \%)$ durante el segundo año y en $1(7,7 \%)$ al tercer año. Aunque las mujeres recayeron más $(37,1 \%$ vs $19,3 \%)$ que los hombres, esta diferen. cia no fue significativa. Tampoco resultaron ser factores significativos, los antecedentes de epilepsia en parientes de primer grado, el tipo de epilepsia, el tipo de crisis, la edad de inicio y el número de crisis previas al tratamiento (Tabla 1 ). A su vez, presentar 2 o más crisis durante el tratamiento, haber recibido una o más curas con medicamentos, aparte de la actual, y haber ingerido 2 o mas medicamentos, son variables significativas en la presentación de las recaídas (Tabla 2).

Tabla 1.

61 niños con epilepsia tratados por 5 años: factores no significativos de recaidas $(\mathrm{p}>0,05)$

\begin{tabular}{|c|c|c|c|}
\hline \multirow[b]{2}{*}{ Factores } & \multirow[b]{2}{*}{ Total } & \multicolumn{2}{|c|}{ Recafidas } \\
\hline & & $N$ & $\%$ \\
\hline Sexo masculino & 26 & 5 & 19,3 \\
\hline Sexo femenino & 35 & 13 & 37,1 \\
\hline \multicolumn{4}{|l|}{ Antecedente } \\
\hline epilepsia 1 grado & 16 & 3 & 18,8 \\
\hline Sin antecedente & 45 & 15 & 33,3 \\
\hline \multicolumn{4}{|l|}{ Epilepsia } \\
\hline idiopática & 45 & 15 & 33,3 \\
\hline \multicolumn{4}{|l|}{ Epilepsia } \\
\hline sintomática & $15^{*}$ & 2 & 13,3 \\
\hline Crisis generalizadas & 52 & 15 & 28,8 \\
\hline Crisis focales & 9 & 3 & 33,3 \\
\hline \multicolumn{4}{|l|}{ Inicjo epilepsia } \\
\hline$<2$ años & 24 & 5 & 20,8 \\
\hline \multicolumn{4}{|l|}{ Inicio epilepsia } \\
\hline$>=2$ años & 37 & 13 & 35,1 \\
\hline \multicolumn{4}{|l|}{$<=2$ crisis } \\
\hline $\begin{array}{l}\text { pre-tratamiento } \\
>=3 \text { crisis }\end{array}$ & 23 & 6 & 30,4 \\
\hline pre-tratamiento & 38 & 11 & 28.9 \\
\hline
\end{tabular}

* un paciente sin datos.

\section{DISCUSION}

Aproximadamente dos tercios de los niños de esta investigación no volvieron a tener crisis y un tercio recayó, proporción muy similar a la comunicada por otros autores (Tabla 3 ). Sin embargo.
Tabla 2.

61 niños con epilepsia tratados por 5 años: factores significativos de recaidas $(p<0,05)$

\begin{tabular}{|c|c|c|c|}
\hline \multirow[b]{2}{*}{ Factores } & \multirow[b]{2}{*}{ Total } & \multicolumn{2}{|c|}{ Recaídas } \\
\hline & & $\mathrm{N}$ & $\%$ \\
\hline $\begin{array}{l}<=1 \text { crisis } \\
\text { durante el } \\
\text { tratamiento } \\
>=2 \text { crisis }\end{array}$ & 47 & 11 & 23,4 \\
\hline $\begin{array}{l}\text { durante el } \\
\text { tratamiento }\end{array}$ & $12^{*}$ & 6 & 50,0 \\
\hline $\begin{array}{l}1 \text { cura con } \\
\text { medicamentos } \\
>=2 \text { curas }\end{array}$ & 43 & 0 & 0,0 \\
\hline $\begin{array}{l}\text { con medicamentos } \\
(17=2: 1=3)\end{array}$ & 18 & 18 & 100,0 \\
\hline $\begin{array}{l}1 \text { medicamento } \\
\text { utilizado } \\
>=2 \text { medica- }\end{array}$ & 49 & 10 & 20,4 \\
\hline mentos utilizados & 12 & 8 & 66,6 \\
\hline
\end{tabular}

* 2 pacientes sin datos.

las muestras no son absolutamente comparables entre sí y su análisis, por la complejidad metodológica del tema, ofrece numerosas dificultades ${ }^{8}$.

Aunque no existe acuerdo al respecto ${ }^{6}$, parece ser que la duración del tratamiento no es un factor de importancia en la aparición de las recaidas. puesto que los enfermos tratados por 2 , $3,4,5$ años y más, recaen en proporción similar ${ }^{7}$, lo cual nos plantea dudas acerca de la necesidad de tratar a nuestros pacientes por períados tan prolongados como 5 años ${ }^{9}$.

El período de observación después del retiro de los medicamentos, es también un hecho que debe ser considerado, pues significa un esfuerzo adicional del paciente con epilepsia en términos de costo y tiempo. Como se aprecia en la Tabla 3 no existe unanimidad entre los autores al respecto, pero es necesario destacar que tiempos prolongados de observación no inciden en aumentar las recaidas 10,11 .

Es generalmente aceptado ${ }^{12,13}$ que el retiro de los anticonvulsivos debe ser hecho en forma gradual, como lo hicimos nosotros en un año. Otros autores disminuyen los fármacos en 2 años $^{14}$ o en 2 ó 3 meses ${ }^{15,16}$ y el porcentaje de reca ídas es semejante (Tabla 3), hecho que también plantea interrogantes acerca de la utilidad de hacer la supresión de la medicación tan lentamente, como en un año o mas.

Alrededor de un tercio de nuestros enfermos recayó durante el periodo de reducción gradual de los fármacos, cifra que puede ser mayor si se analizan otras series publicadas ${ }^{10}$. El análisis de estas "crisis de retirada"9 obliga a preguntarse si 
Tabla 3.

Recaidas después de suprimir la medicación en niños con epilepsia

\begin{tabular}{lcccrc}
\hline Autores & Año & $\begin{array}{c}\text { Duración } \\
\text { terapia } \\
\text { (Años) }\end{array}$ & $\begin{array}{c}\text { Obsetvación } \\
\text { post-supre- } \\
\text { sión (Años) }\end{array}$ & N & $\%$ \\
\hline Hollowach y cols. & 1972 & 4 & $5-12$ & $36 / 148$ & 24,3 \\
Groh & 1975 & 3 & 3 & $152 / 565$ & 26,9 \\
Oller - Daurella y cols. & 1977 & +5 & +10 & $69 / 228$ & 30,3 \\
Rodin y John & 1980 & 3 & 2 & $2 / 32$ & 6,2 \\
Todt & 1981 & $2-4$ & 3 & $159 / 433$ & 36,7 \\
Hollowach & 1982 & 4 & $15-23$ & $41 / 148$ & 27,7 \\
Emerson y cols. & 1982 & 4 & 2,7 & $18 / 68$ & 26,4 \\
Janz y cols. & 1982 & 2 & 2 & $25 / 59$ & 42,4 \\
Shinnar y cols. & 1985 & 2 & 2 & $22 / 68$ & 25,0 \\
Devilat y cols. & 1986 & 5 & 2 & $18 / 61$ & 29,5 \\
\hline
\end{tabular}

ellas representan un fenomeno similar al que ocurre con el retiro de sedantes de acción corta, como el alcohol, barbitúricos y benzodiazepinas ${ }^{17,18}$ y si así fuera, la reintroducción del tratamiento anticonvulsivo después de las "crisis de retirada" no estaría indicada: Sin embargo, es imposible responder a esta cuestión por ahora ${ }^{8}$.

Siendo una realidad que algunos pacientes recaen, otros no y que los factores hasta aquí mencionados no permiten predecir qué evolución clínica tendrâ la enfermedad, el estudio de otras variables podría ser una ayuda eficaz en este sentido ${ }^{7}$.

El sexo y los antecedentes de epilepsia en la familia no fueron predictores de recaídas, to cual ha sido demostrado consistentemente por otros autores $10,11,15,19$, salvo formas especiales de crisis epilépticas 20 .

Tampoco hubo más recaídas entre los pacientes con daño pre-peri o postnatal, que en aquellos que no los presentaron. Se ha demostrado que los enfermos orgánicos recaen significativamente más que Jos que no $\operatorname{son}^{11.13}$, pero dentro de ese concepto, los investigadores mencionados incluyeron además de los antecedentes personales, el retardo menfal y daños de primera neurona, últimas dos caracteristicas que sólo poseia un escaso número de nuestros pacientes, por to que una comparación con dichas experiencias es imposible. Sin embargo, otras publicaciones ${ }^{\mathbf{4}}$ no han podido confirmar que el daño orgánico sea realmente un factor predictivo de recaídas.

No existe acuerdo acerca de si el tipo de crisis sea una buena variable de predicción. Nuestra investigación no demostró diferencias en las recaídas entre pacientes con crisis generalizadas o focales. Es cierto que nuestros enfermos son pocos y que algunos que presentaban crisis focales también tenían crisis generalizadas, pero los mismos hallazgos han sido comunicados por otros autores ${ }^{15}$. Sin embargo, las crisis jacksonianas $^{11}$ y las parciales complejas ${ }^{11,16}$ parecieran tener especial tendencia a las recaídas.

La jniciación de la epilepsia antes de los dos años de vida no fue un factor significativo de mal pronóstico en nuestros pacientes. Aunque una investigación haya demostrado lo contrario ${ }^{\mathbf{1 5}}$, otros trabajos confirman nuestros resultados en nifios $\mathrm{s}^{11,14,15}$ y adultos 21,22 .

La severidad de las crisis epilépticas, es decir, la frecuencia de ellas antes de iniciar el tratamiento, es un factor importante de recaídas en adul$\operatorname{tos}^{8}$ y niños ${ }^{15}$, sin embargo, no existe total acuerdo al respecto, por cuanto en nuestra investigación y en otras ${ }^{14,11}$, ello no ha podido ser demostrado. Desde otro punto de vista, se sabe que las recaídas, durante el tratamiento no son muy frecuentes, aunque dependen del tipo epilepsia $23,24, y$ que la mayoria de ellas ocurre precozmente 24 .

Como ha sido demostrado en nuestra investigación, los pacientes que presentacon 20 más episodios mientras estaban recibiendo medicación, recayeron cuando ésta fue suspendida, más frecuentemente que aquellos en los cuales la enfermedad es controlada desde el inicio del tratamiento, lo que también ha sido comunicado por otros autores 11,15 .

Los enfermos que requirieron más de una droga anticonvulsiva para el control de su epilepsia, recayeron significativamente más que los que necesitaron una sola, hecho que se relaciona con peor pronóstico de la enfermedad y que también ha sido observado por otros autores ${ }^{15,25}$. Todos los pacientes que habían recibido uno o más períados de tratamiento previo con drogas anticonvulsivas por un tiempo de 5 años, recayeron; en cambio esto no ocurrió en ninguno de los que 
habían recibido por primera ve $z$ dichos fármacos, lo que indica nuevamente que la severidad de la enfermedad puede ser un importante factor de pronóstico $8,15.24$ y plantea dudas acerca de la conveniencia de retirar la medicación en enfermos a quienes se les ha indicado una segunda cura con anticonvulsivantes. Sin embargo, no hay estudios con casuísticas numerosas en pacientes con las características seinaladas ${ }^{8}$, por lo que nuestros hallazgos deberían ser comprobados en futuras investigaciones.

Aunque la muestra estudiada es muy homogénea, de los resultados expuestos pareciera poder concluirse que existirian pacientes portadores de una mala evolución desde el inicio de] tratamiento o durante él, como sucedió con nuestros 18 niños que recayeron. Sin embargo, no nos fue posible identificar factores que pudieran haber predicho las recaidas, aparte de las que dicen relación con la evolución de la enfermedad, así, se podría pensar en la existencia de "epilepsias tratables" 26 , con favorable evolución ${ }^{27}$ y otras con un curso desfavorable, relacionadas talvez, con algún factor genético.

Por otra parte, existen enfermos que en estudios de análisis factoriales uni o multivariados $11,15,16$, demuestran tener ciertas caracteristicas que los hacen más vulnerables a las recaidas y otros pacientes que no las tienen. Esto es importante por dos razones: primero, por quc aquellos enfermos con mayores riesgos deberían tratarse por periodos más prolongados que aquellos que no los tienen ${ }^{9}$ y segundo. porque siendo la epilepsia un síndrome que incluye numerosos cuadros diferentes $s^{26,28}$, los estudios sobre evolución y pronóstico deberían ser realizados considerando las diversas formas de expresión de la enfermedad, para lo cual se requerirán estudios colaborativos que incluyan casuísticas más numerosas ${ }^{8}$.

\section{RESUMEN}

Para estimar la frecuencia de recaídas y los factores que podrían influir en ella, se estudiaron 61 niños con epilepsia que habian sido tratados por 5 años. libres de crisis, y observados por lo menos durante 2 años después de suspendidos los medicamentos. En 29,5\% de los pacientes se registraron recaidas, la mayoría de ellas dentro de los dos primeros años desde que se inició el retiro de los fármacos. Las recaídas se relacionaron significativamente con mala evolución duran. te el tratamiento, ingestión de 2 o mas drogas anticonvulsivas, más de una cura de 5 años con medicamentos antes de la actual. No se encontraron relaciones significativas con sexo, antecedentes familiares de epilepsia, tipo de crisis, edad de inicio y número de crisis preyias al trata. miento.

\section{REFERENCIAS}

1. Sofijonov, N.G.: Clinical evolution and prognosis of childhood epilepsies. Epitepsia 23: 61, 1982.

2. Hirtz, D.G., Ellenberg, H.H. and Nelson, K.B.: The risk of recurrence of nonfebrile seizures in children. Neurology (Cleveland) 34:637, 1984

3. Devilat, M., Menéndez, P., Chamorro, R. y Acevedo. $C$ : Seguimiento de nî̃os con una crisis epiléptica no tratada. Rev. Clil. Pediatr. 55: 9, 1984.

4. Devilat, $M, y$ Menéndez, P.: La oligoepilepsia. Una manera diferente de ser epiléptico? Rev. Chil. Pcdiatr. 56: 53, 1985.

5. Reynolds, E.H.: Chronic antiepileptic toxicity. A review. Epilepsì 16: 319, 1975.

6. Devilat, M. Germain, L. y V'alenzaela, B.: Pronóstico a largo plazo en niños con crisis epilépticas tratadas por 5 años. Rev. Chil. Pediatr. 54: 319 , 1983.

7. Devilat, M., Germain, L. y Valenzuela, B.: Factores de predicción de recidivas en niños con crisis epilépticas tratadas por 5 años. Rev. Chil. Pediatr. 54 : $396,1983$.

8. Chadwick, D.: The discontinuation of antiepileptic therapy. In: Pedley, T.A. and Meldrum, B.S. (eds.): Recent Advances in Epilepsy. Churchill Livingstone, New York, 1985, pp. 111-124.

9. Gordon, N.: Duration of treatment for childhood epilepsy. Develop. Med. Child, Neurol. 24: 84, 1982.

I0. Hollowach, J., Thusston, D.L. and O'Leary, J. Prognosis in childhood epilepsy. Follow-up study of 148 cases in wich therapy had been suspended after prolonged anticonvulsant control. New Engl. J. Med. 286: 169,1972 .

I 1. Hollowach, J., Thruston, D.L., Hixon, B.B. and Keller, A.J.: Prognosis in childhood epilepsy. Additional follow-up of 148 children 15 to 23 years afte: withdrawal of anticonvulsant therapy. New Engl. J. Med. 306 : 831, 1982.

12. Degen, R.: Die kindlichen Anfallsleiden. Hippokrates Verlag, Stuttgart, 1976, S. 139.

13. Matthes, A.: Epilepsien. Thieme Vertag, Stuttgatt, 1984, S. 235.

14. Groh, C.: Zur Frage der Heilbarkeit kindlicher Epilepsien. Wien Klin. Wochenschr. 87: 1, 1975.

15. Kmerson, R, D'Souza, B.J., Vining, E.P., Holden, K.R., Mellits, E.D. and Fredmon, J.M.: Stopping medication in children with epilepsy. Predictors of outcome. New Engl. J. Med. 304: 1125, 1981.

16. Shimar, S, Vining, E.P., Mallits, E.D., D'Souza,

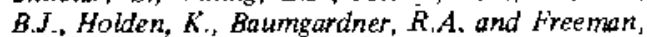
J.M.: Discontinuing antiepileptic medication in children with epilepsy af ter two years without seizures. New Eng1. J. Med. 313: 976, 1985.

17. Victor, M.: The role of alcohol in the production of seizures. In: Modern Problems in Pharmacopsychiatry, Karges, Basel, 1970, pp. 185.

18. Wikler, A. and Essig, C.F.: Withdrawal seizures following clronic intoxication with barbiturates and other sedative drugs. In: Wodern Problems in Pharmacopsychiatry, Karger, Basel, 1970, pp. 170.

19. Janz, D., Kern, A., Mossinger, H.J. and Puhimonn. $U_{\text {.: }}$ Ruckfall-Prognose nach Reduktion der Medikamente bei Epilepsiebehandlung. Nervenarzt 54: 525,1983 . 
20. Devilat, M. y Germain, L.: Pequeño mal impulsivo. Síndrome de Janz. Rev, Chil, Pediatr, 54: 11, 1982.

21. Juul-Jensen, $P$.: Frecuency of recurrence after discontinuance of antionvulart therapy in patients with epileptic seizures. Epilepsia 5: 532, 1964.

22. Annegers, J.F., Houser, W.A. and Elveback, L.R.: Remission of seizures and replapse in patients with epilepsy. Epilepsia 20: 729, 1979.

23. Devikat, M. y Taho, L.: Epilepsia infantil criptogenética. Pediatria (Santiago) $21 \div 204,1978$.
24. Shorvon, S.D. and Reynolds, E.H.: Early prognosis of epilepsy. Brit. Med. J. 285: 1699, 1982.

25. Cabrera, F., Acevedo, C. y Mugoz, M.: Politerapia en epilepsias. Rev. Chil. Pediatr. 55: 253, 1984.

26. Delgado-Escueta, A.V., Treiman, D.M. and Walsh, G.O.: The treatable epilepsies. New Engl. J. Med. 308: 1508, 1983.

27. Zielinski, $J J .:$ Epileptics not in treatment. Epilepsia 15: 203, 1974

28. Gómex, M.R.: Epilepsies of infancy and childhood. Ann. Neurol. 13: 113, 1983. 